\title{
Use of fluorescence quantitative polymerase chain reaction (PCR) for the detection of Escherichia coli adhesion to pig intestinal epithelial cells
}

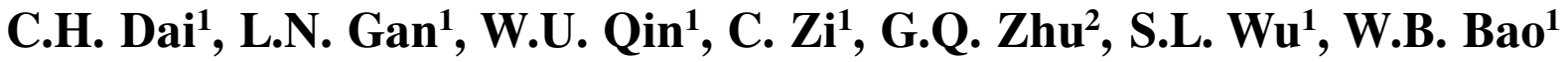 \\ ${ }^{1}$ College of Animal Science and Technology, Key Laboratory for Animal Genetics, \\ Breeding, Reproduction and Molecular Design of Jiangsu Province, Yangzhou University, Yangzhou, China \\ ${ }^{2}$ College of Veterinary Medicine, Yangzhou University, Yangzhou, Jiangsu, China
}

\begin{abstract}
An efficient and accurate method to test Escherichia coli (E. coli) adhesion to intestinal epithelial cells will contribute to the study of bacterial pathogenesis and the function of genes that encode receptors related to adhesion. This study used the quantitative real-time polymerase chain reaction (qPCR) method. qPCR primers were designed from the PILIN gene of E. coli F18ab, F18ac, and K88ac, and the pig $\beta$-ACTIN gene. Total deoxyribonucleic acid (DNA) from E. coli and intestinal epithelial cells (IPEC-J2 cells) were used as templates for qPCR. The $2^{-\Delta \Delta C t}$ formula was used to calculate the relative number of bacteria in cultures of different areas. We found that the relative numbers of F18ab, F18ac, and K88ac that adhered to IPEC-J2 cells did not differ significantly in 6-, 12-, and 24-well culture plates. This finding indicated that there was no relationship between the relative adhesion number of $E$. coli and the area of cells, so the method of qPCR could accurately test the relative number of $E$. coli. This study provided a convenient and reliable testing method for experiments involving E. coli adhesion, and also provided innovative ideas for similar detection methods.
\end{abstract}

Key words: Adhesion, Escherichia coli, intestinal epithelial cells, pig, quantitative real-time PCR

\section{Introduction}

Enterotoxigenic Escherichia coli (ETEC), one of the main pathogens that cause diarrhea in piglets, which seriously threatens the economic benefits of scale pig farms with high mortality (Turner et al. 2006, Zhang et al. 2007). The pathogenic Escherichia coli (E. coli) can adhere to pig intestinal epithelial cells
(IPEC-J2 cells) in a process mediated by fimbriae, then secrete enterotoxin and ultimately lead to edema and diarrhea in piglets (Bertschinger et al. 1972, Hohmann et al. 1975, Imberechtset al. 1994). Common enterotoxigenic E. coli bacillosis in scale pig farms are mainly caused by F18 and K88. Studies showed that whether they could cause disease in piglets depended upon the existence of specific receptors for adhesion 
Table 1. Primer sequences of genes analyzed by real-time PCR.

\begin{tabular}{ccc}
\hline Primer name & Primer sequences (5'-3') & Product length (bp) \\
\hline$P I L I N-\mathrm{F}$ & AGGCCGAACCAAAGAAGCAT & 117 \\
\hline$P I L I N-\mathrm{R}$ & TCACCATCAGGGTTTCTGAGT & 119 \\
\hline$\beta-A C T I N-\mathrm{F}$ & GTCGTACTCCTGCTTGCTGAT & \\
\hline$\beta-A C T I N-\mathrm{R}$ & CCTTCTCCTTCCAGATCATCGC & \\
\hline
\end{tabular}

in small intestinal mucosal epithelial cells in piglets (Nataro and Kaper 1998, Melkebeek et al. 2012). Therefore, the pathogenicity of $E$. coli is mainly studied by detecting its ability to adhere to host intestinal epithelial cells from the perspective of bacteria (Yu et al. 2012). Also, the functions of receptor related genes of $E$. coli can be analyzed by analyzing the adhesion ability of intestinal epithelial cells to $E$. coli from the perspective of the host to reveal the role of these genes regarding resistance to $E$. coli colonization (Wu et al. 2007). Thus, it can be proposed that it is particularly important to analyze and detect $E$. coli adherence to host intestinal epithelial cells, for which studying not only the pathogenicity of ETEC but also the mechanism of host resistance to $E$. coli.

Currently, there are main three types of detection methods to assess the ability of $E$. coli to adhere to host intestinal epithelial cells. First, the number of adherent bacteria can be estimated based on the number of bacteria colonies obtained in a plate coated with collected bacteria liquid ( $\mathrm{Li}$ et al. 2010, Yu et al. 2012, Tawakoli et al. 2013, Shi et al. 2015). Second, the number of adherent bacteria in a unit view on a glass slide under a microscope can be observed (Zhang et al. 2010, Wu et al. 2014). Third, the number of bacteria can be detected by the absolute quantitative method by estimating the unit cell number (Candela et al. 2008, Atshan et al. 2012). However, there are several limitations in above-mentioned three methods because it is not easy to guarantee the actual quantity of adherent bacteria and to control a consistent culture density of cells with different treatments.

To establish an efficient and accurate method for detecting the adhesion ability of $E$. coli to small intestinal epithelial cells in pigs, a relative quantitative method was used in this present study. We used inherent gene $\beta$-ACTIN expressed stably in IPEC-J2 cells as an internal reference and used fimbria protein gene PILIN of E. coli as target gene. Then we used total DNA extracted from cells with bacteria adhesion as templates to obtain the relative amount of bacteria adhesion to cells by detecting the copies of PILIN gene of $E$. coli compared to inherent gene $\beta$-ACTIN expressed in IPEC-J2 cells, which provided a convenient and reliable detection method for adhesion-related experiments for ETEC.

\section{Materials and Methods}

\section{Experimental materials and reagents}

A pig intestinal epithelial cell line (IPEC-J2) was obtained from the University of Pennsylvania (Philadelphia, USA). E. coli F18ab, E. coli F18ac, and E. coli K88ac, which were standard strain of Escherichia coli from Culture Collection Centers in the United States (ATCC), were provided by Professor Zhu Guoqiang of Veterinary College, Yangzhou University (Yangzhou, China). Dulbecco's modified Eagle's medium (DMEM), F-12 Nutrient Mixture medium, and fetal bovine serum (FBS) were purchased from Gibco (New York, USA). Yeast powder and peptone were purchased from Thermo Fisher Oxoid (Basingstoke, UK). The DNA extraction kit (DP304) and PCR product purification kit (DP214) were purchased from Tian Gen Biotechnology Beijing Co. (Beijing, China). The Qtaq ${ }^{\mathrm{TM}}$ One-Step qRT-PCR SYBR ${ }^{\circledR}$ KIT (639519) was purchased from Takara Biotechnology Dalian Co. (Dalian, China).

\section{Primer design and PCR amplification}

Using Primer Express 2.0 software, primers for the gene encoding the fimbria protein, PILIN, in $E$. coli was designed based on the sequence M25302.1 (http://www.ncbi.nlm.nih.gov/) in GenBank, and primers for the $\beta$-ACTIN gene in pigs were designed based on the sequence NC_010445.3 (http://www.ncbi.nlm.nih.gov/) in GenBank; both primer pairs were synthesized by Takara Biotechnology Dalian Co. (China). Primer sequences to amplify PILIN and $\beta$-ACTIN are listed in Table 1.

We obtained DNA extracted from bacteria and cells with a DNA extraction kit as a template for PCR amplification reactions. The $20-\mu \mathrm{L}$ PCR reaction mixture contained the following: $1 \mu \mathrm{L}$ DNA (100 ng), $0.4 \mu \mathrm{L}$ forward primer $(10 \mu \mathrm{M}), 0.4 \mu \mathrm{L}$ reverse primer $(10 \mu \mathrm{M}), 10 \mu \mathrm{L}$ SYBR Premix ExTap ${ }^{\mathrm{TM}}$ II $(2 \times)$, and $8.2 \mu \mathrm{L}$ RNase-free $\mathrm{H}_{2} \mathrm{O}$. PCR reaction conditions were as follows: $95^{\circ} \mathrm{C}$ for $30 \mathrm{~s}$, followed by 40 cycles of $95^{\circ} \mathrm{C}$ for $5 \mathrm{~s}$ and $60^{\circ} \mathrm{C}$ for $34 \mathrm{~s}$, and a final hold at $4^{\circ} \mathrm{C}$. PCR amplification products were detected by $10 \%$ 
polyacrylamide gel electrophoresis, and the sequences of amplified fragments that were detected by direct sequencing were compared with gene sequences under conditions for which the length of the amplified fragments were similar to those of the target fragments.

\section{Formulation of a standard curve for qPCR}

PCR products were purified and recovered before gradient dilution by 10 -fold increments, which were diluted to recovery solution concentrations of $10^{-1}, 10^{-2}, 10^{-3}, 10^{-4}, 10^{-5}$, and $10^{-6}$. QPCR amplification was performed in a $20-\mu \mathrm{L}$ reaction mixture containing $2 \mu \mathrm{L}$ DNA (200 ng), $0.4 \mu \mathrm{L}$ of $10 \mu \mathrm{M}$ each forward and reverse primer, $0.4 \mu \mathrm{L} 50 \times$ ROX Reference Dye II, $10 \mu \mathrm{L} 2 \times$ SYBR Green SYBR Premix ExTaq $^{\mathrm{TM}} \mathrm{II}$, and $6.8 \mu \mathrm{L}$ RNase-free $\mathrm{H}_{2} \mathrm{O}$. PCR reaction conditions were as follows: $95^{\circ} \mathrm{C}$ for $15 \mathrm{~s}$, followed by 40 cycles of $95^{\circ} \mathrm{C}$ for $5 \mathrm{~s}$ and $62^{\circ} \mathrm{C}$ for $34 \mathrm{~s}$. Analysis of the melting curve was carried out after PCR amplification to determine the specificity of the amplified products. Melting curve program were as follows: $95^{\circ} \mathrm{C}$ for $15 \mathrm{~s}, 62^{\circ} \mathrm{C}$ for $60 \mathrm{~s}, 95^{\circ} \mathrm{C}$ for $15 \mathrm{~s}$, and $60^{\circ} \mathrm{C}$ for $15 \mathrm{~s}$. The standard curve was analyzed after amplification by selecting the standard curve running mode.

\section{DNA extraction after $E$. coli adhesion to IPEC cells}

We want to detect the unit number of cells with bacteria adhesion in this study. In order to eliminate different number of the cultured cell's impact on the number of bacteria adhesion, we use different specifications of the cell culture plates to culture the corresponding amount of cells. Considering the suitable density of cell culture and make it facilitate our homogenization treatment in the process of the experiment, intestinal epithelial cells were inoculated to 6 -well plates at a density of $1.2 \times 10^{6}$ cells per well, 12 -well plates at a density of $5.0 \times 10^{5}$ cells per well, and 24-well plates at a density of $2.5 \times 10^{6}$ cells per well. Intestinal epithelial cells were incubated for 12 $\mathrm{h}$ in an incubator under the conditions of $37^{\circ} \mathrm{C}$ and $5 \% \mathrm{CO}_{2}$. E. coli F18ab, F18ac, and K88ac were each inoculated to LB culture medium and incubated for $12 \mathrm{~h}$ at a rotating speed of $200 \mathrm{rev} / \mathrm{min}$ on a shaking table. PBS buffer solution was used for resuspension, wash, and centrifuge the pellet collected after centrifugation for $10 \mathrm{~min}$ at a rotating speed of 3000 $\mathrm{rev} / \mathrm{min}$. The above operation was repeated three times. Cell culture medium was used to dilute $E$. coli to $1.0 \times 10^{9} \mathrm{CFU} / \mathrm{mL}$ and $2.5,1.0$, or $0.5 \mathrm{~mL}$ bacterial suspensions were added to 6-, 12-, and 24-well culture plates, respectively, and each experiment was repeated three times. Cells with different treatments were incubated for $1 \mathrm{~h}$ in an incubator under the conditions of $37^{\circ} \mathrm{C}$ and $5 \% \mathrm{CO}_{2}$. The bacterial liquid in the cell culture plates was discarded and cells were washed three times with PBS buffer solution, then DNA lysis solution was added to cell culture plates to extract total DNA of cells and bacteria following the kit manufacturer's instructions.

\section{Quantitative determination and statistical analysis}

We took mixed DNA of cells and bacteria as templates for triplicate reactions, and the relative quantitative method was used to detect DNA amplification. The $2^{-\Delta \Delta \mathrm{Ct}}$ method was used to process the real-time PCR results (Livak and Schmittgen 2001). Expression of the $\beta$-ACTIN gene in cells was used to normalize the measurements of the gene encoding the fimbria protein, PILIN, for which $\Delta \mathrm{Ct}=$ the $\mathrm{Ct}$ values of bacteria - the $\mathrm{Ct}$ values of cells, and $\Delta \Delta \mathrm{Ct}=$ the $\Delta \mathrm{Ct}$ values of experimental group (the $\Delta \mathrm{Ct}$ values of PILIN gene in each kind of bacteria, respectively) - the $\Delta \mathrm{Ct}$ values of reference group (the average of $\triangle \mathrm{Ct}$ values of PILIN gene for three kinds of bacteria). Statistical analyses were carried out using the General Linear Model (GLM) of SPSS 16.0 software (SPSS Inc., Chicago, IL, USA) to compare and analyze the relative copies of bacterial genes among culture plates with different areas.

\section{Results}

\section{Identification of PCR amplification products}

PCR amplification products were detected by $10 \%$ polyacrylamide gel electrophoresis (PAGE). As shown in Fig. 1, the sizes of the electrophoresis bands were consistent with those of the target fragments. PCR amplification products were detected by sequencing and compared with gene sequences (Supplementary Fig. 1). Our findings showed that amplified sequences from $E$. coli F18ab, F18ac, and K88ac were consistent with that of the PILIN gene sequence, which indicated that the target gene fragments were specifically amplified and that the gene had a high degree of conservation among different E. coli serotypes. Therefore, these primers can be used for real-time fluorescence quantitative detection. 


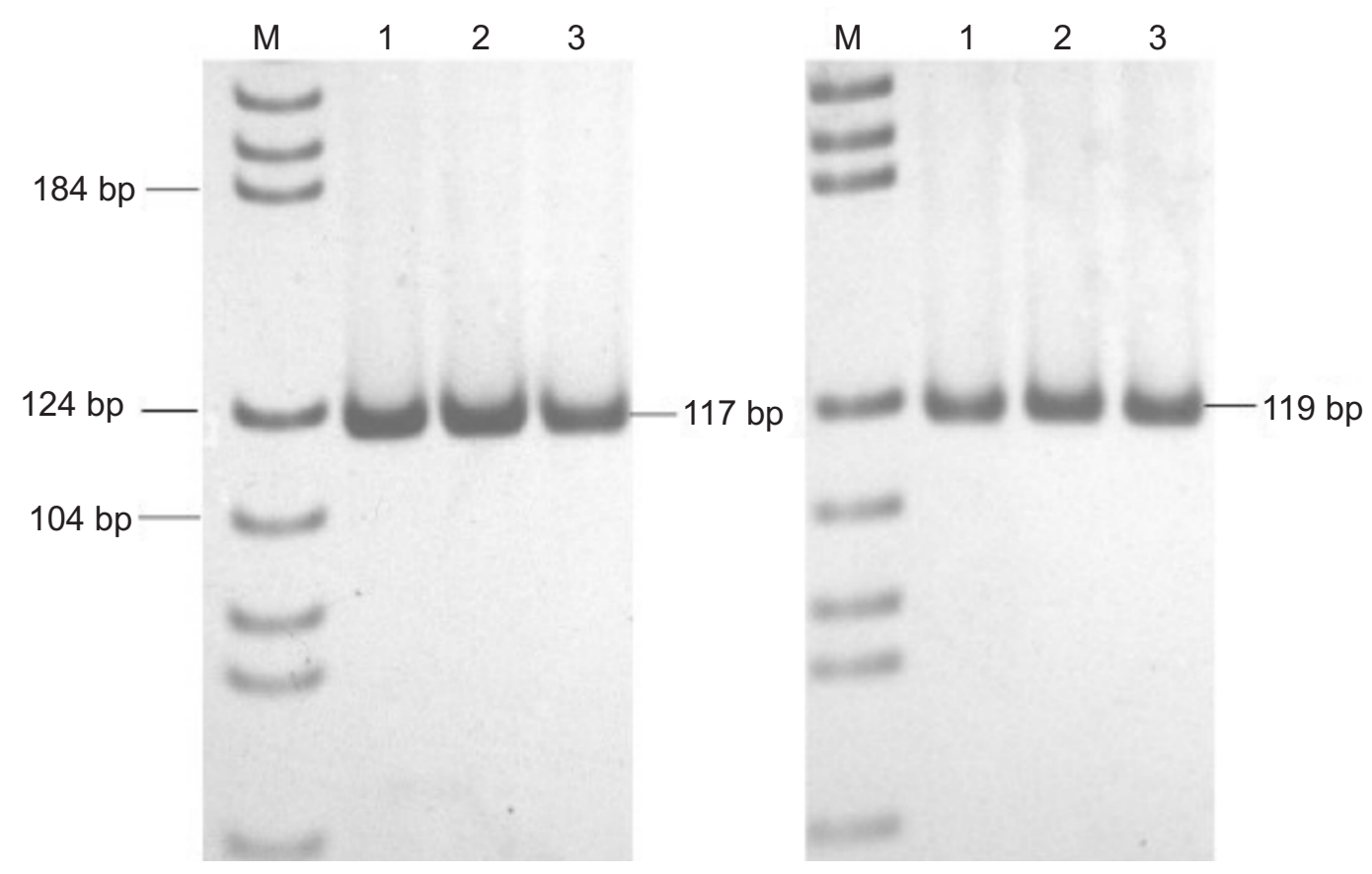

Fig. 1. Detection of PCR products by polyacrylamide gel electrophoresis. The left part represents PILIN gene and the right represents $\beta$-ACTIN gene, M represents $\mathrm{pBR} 322 / \mathrm{Msp}$ I DNA Marker and lanes 1, 2, and 3 represent the PCR products from the total DNA of cells and bacteria after E. coli adhesion experiment by F18ab, F18ac, and K88ac, respectively.

Supplementary Fig. 1. The sequencing results of PCR products of PILIN gene. They were blasted with the sequence of PILIN gene in NCBI database.

\section{Analysis of amplification curve, standard curve and melting curve}

The results of the PCR amplification curve, standard curve and melting curve obtained by real-time fluorescent quantitative PCR are shown as Figures 2 and Figure 3, which indicated that the amplification curves for the PILIN and $\beta$-ACTIN genes were smooth and there was obvious exponential growth. The linear correlation coefficient of the amplification curves for the PILIN gene reached 1, and those of the $\beta$-ACTIN gene reached 0.999 , which illustrated that the standard curves were accurate and reliable. Concurrently, the amplification efficiency of the PILIN gene was $96.999 \%$ and that of the $\beta-A C T I N$ gene was $99.635 \%$, which indicated that the internal and target gene amplification efficiencies were suitable for the $2^{-\Delta \Delta \mathrm{Ct}}$ method of calculation.

Expression of the $\beta-A C T I N$ gene in cell lines was used as a reference to normalize the amount of expression of the fimbria gene PILIN, and the average of the $\Delta \mathrm{Ct}$ values in different cell culture plates (6-, 12-, and 24-well) was used as a reference group to calculate the copies of the fimbria gene compared with that of the reference group by the $2^{-\Delta \Delta \mathrm{Ct}}$ method. The relative copies of the bacterial gene could be equated with the relative adhesion level of bacteria because it was directly related to the number of bac- teria. As shown in Figures 4, there were no significant differences in the adhesion levels of E. coli F18ab, F18ac, or K88ac to IPEC-J2 cells with different culture areas. Additionally, the numbers of adherent bacteria in a unit area of cells were similar, which illustrated the accuracy of detecting adhesion to intestinal epithelial cells in pigs through the method of relative quantitative PCR established in this present study.

\section{Discussion}

The basic principle for detecting the transcriptional expression levels of genes by relative quantitative PCR is that there is a linear relationship between the amplification cycles ( $\mathrm{Ct}$ values) when the measured fluorescence signal reaches a threshold equal to the original copy number of the template. Thus, the original copy number of specific genes can be influenced by the sample concentration. When selecting one or more housekeeping genes as a reference, an ideal gene should maintain an active transcriptional state in all cells, show less variation in different stages and tissues during growth and development, and be essential to maintain the basic physiological requirements of the body. So, we can analyze mRNA expression changes in target genes by comparing the copies of target genes (such as PILIN gene in this study) 


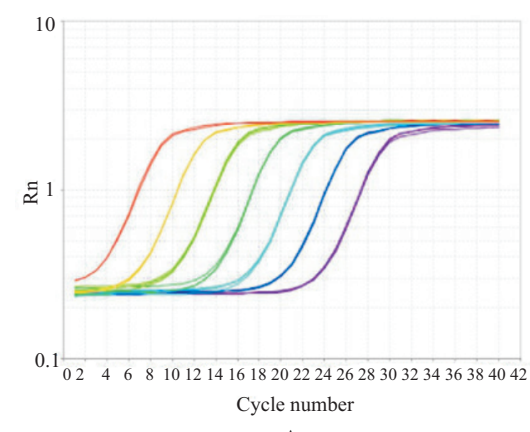

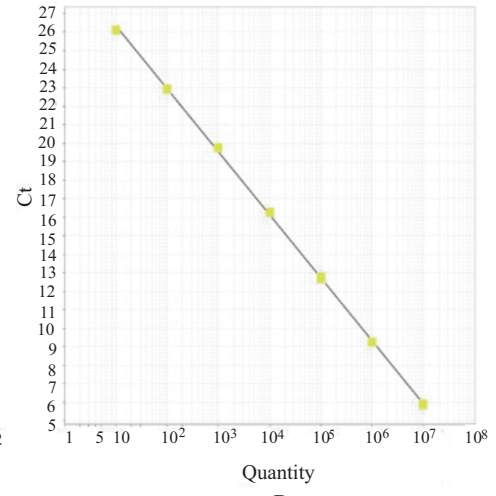

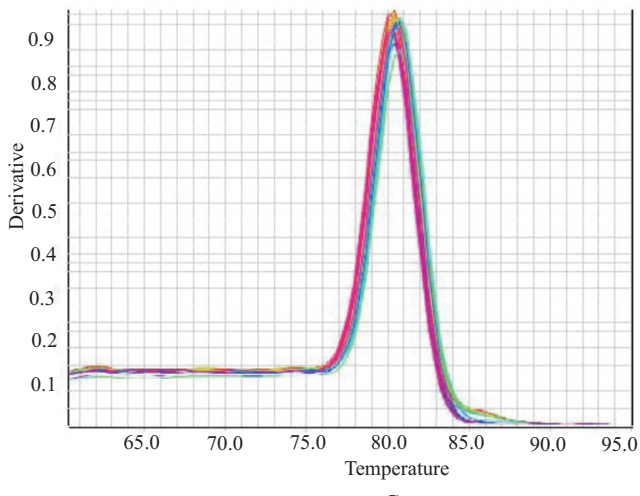

C

Fig. 2. Quantitative PCR amplification curve, quantitative PCR standard curve and melting curve of the PILIN gene. A represents quantitative PCR amplification curve; B represents quantitative PCR standard curve (Slope: -3.396; R²: 1; Eff \%: 96.999); $\mathrm{C}$ represents melting curve.

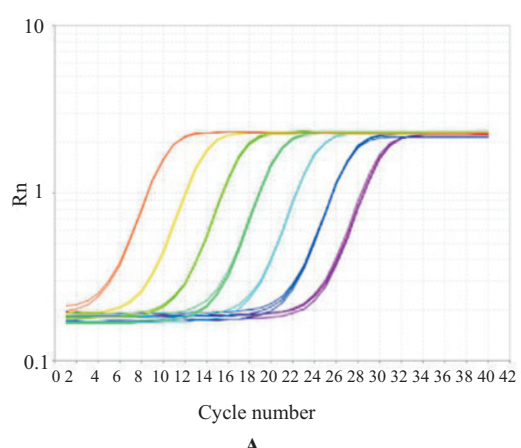

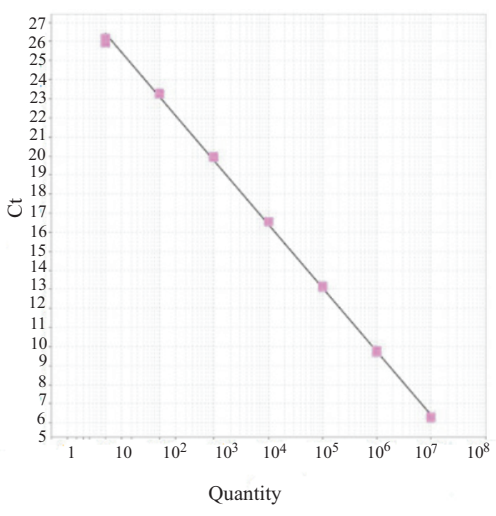

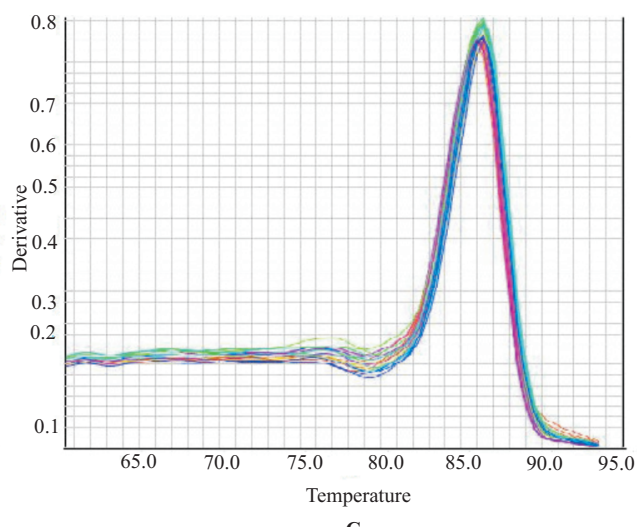

Fig. 3. Quantitative PCR amplification curve, quantitative PCR standard curve and melting curve of the $\beta$-ACTIN gene. A represents quantitative PCR amplification curve; B represents quantitative PCR standard curve (Slope: -3.331 ; $\mathrm{R}^{2}$ : 0.999; Eff \%: 99.635); C represents melting curve.
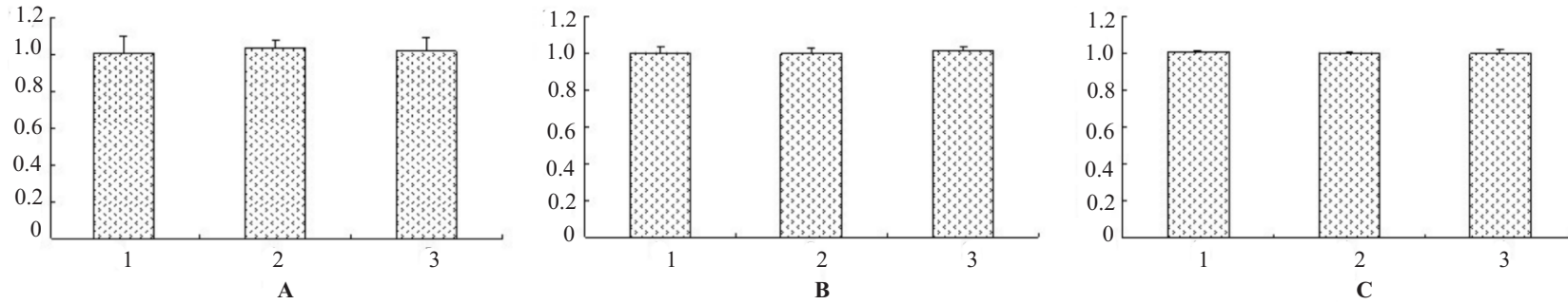

Fig. 4. Adhesion level of E. coli F18ab, F18ac and K88ac in IPEC-J2 with different culture areas. A, B and C represents respectively F18ab, F18ac and K88ac; 1, 2, and 3 represents respectively the test results of 6-, 12-, and 24-well culture plates.

to those of housekeeping genes (also known as reference genes, such as $\beta$-ACTIN gene in this study) with different treatments and different growth stages or organizations. This study relied upon the basic principle of relative quantitative PCR to detect and analyze copies of bacterial genes relative to the copies of cellular genes in pigs, in which we used total DNA collected after bacteria adhesion as a template, a cellular gene as an internal gene, and a bacterial gene as a target gene. Because the gene copies were proportional to the number of bacteria or cells, the relative copies of bacterial genes could be equated to the adhesion level of bacteria. Importantly, the relative quantitative detection data could be expressed as the relative adhesion level of bacteria. It is feasible that this study could yield insights into the detection of relative amounts of bacterial adhesion to cells. 
Compared with other commonly used methods, the qPCR protocol established in this study to detect the adhesion level of bacteria has the obvious advantages in collection of the samples and ease of detection by PCR. Cell lysate solution was added immediately to the cell culture plates after incubating for $1 \mathrm{~h}$ and washing with PBS buffer solution, which could rapidly terminate the proliferation of bacteria, thereby excluding any effect on the accuracy of adhesion detection caused by proliferating bacteria. Additionally, another very important advantage is that data obtained using the relative quantitative method in this experiment can be considered to indicate the relative number of bacteria that adhere to each cell. Therefore, there is no high requirement for the cell growth density of different treatment groups when detecting bacterial adhesion levels to cells with different treatments. When analyzing and verifying the function of genes related to resistance to $E$. coli at the cellular level, we need to intervene on many levels such as gene sequences, expression level, and function level, to explore the relationship between gene function and bacterial adhesion, which could inevitably lead to differences in the cellular state and growth rate. Ultimately, the detection method used in this experiment could yield satisfactory results without considering the density of cell growth or culture area. For example, when verifying the function of target genes by new technologies, such as RNA interference (RNAi) and gene knockout, we can use this type of method to detect differences in bacterial adhesion to host cells after gene silencing and gene modifying without considering the differences in the growth rate of cells caused by the interference vector or the screening process used to generate a knockout. Additionally, the quantitative PCR method can be operated under a fully closed environment, which has the advantages of high specificity, high sensitivity, good repeatability, speed, and accuracy, allowing it to best guarantee the accuracy of test results (Schmittgen 2001, Wall and Edward 2002). We calculated that the amplification efficiency of the PILIN gene was $96.999 \%$ and that of the $\beta$-ACTIN gene was $99.635 \%$ by drawing standard curves, and they had a good linear correlation. So, the quantitative primers designed in this study had good specificity and their amplification efficiency was close to $100 \%$, which can be calculated relative to the quantitative amount with the formula of $2^{-\Delta \Delta \mathrm{Ct}}$ (Livak and Schmittgen 2001).

In this study, E. coli F18ab, F18ac, and K88ac were used to adhere to the intestinal epithelial cell line with different culture areas to detect the reliability of detecting relative adhesion by this quantitative method. The detection method of relative quantitative PCR can reveal the number of adherent bacteria on the unit number of cells. This experiment proved that the detection results were independent of the number of cells through the use of different cell culture plates. The fimbria gene sequence was used as a template to design quantitative PCR. We found that the amplified fimbria gene sequence had strong conservation by comparing it with the sequencing results available in public databases. Thus, the quantitative PCR primers designed in this study can be widely used in the verification of adhesion by various bacteria. Moreover, the specific quantitative PCR primers can be used for the detection of adhesion by different types of bacteria according to this method, and which can also provide a method for the study of levels of bacterial colonization in vivo.

In conclusion, the quantitative PCR primers that we designed could completely meet the requirements of detection, which based on the relative adhesion results further confirmed that the method is exact and reliable. The real-time fluorescent quantitative method designed in this study for detecting $E$. coli adhesion to intestinal epithelial cells has not only the advantages of being feasible, accurate, efficient, and convenient, but also provides a new way of thinking for the research in detecting the number of $E$. coli bacteria adhering to the intestine in vivo.

\section{Acknowledgments}

This research was supported by the National Natural Science Funds (31572360, 31372285), Genetically Modified Organisms Technology Major Project (2014ZX08006-001B), Science and Technology Supporting Project of Jiangsu Province (BE2015329, BE2014357), and the Priority Academic Program Development of Jiangsu Higher Education Institutions (PAPD).

\section{References}

Atshan SS, Nor Shamsudin M, Sekawi Z, Lung LT, Hamat RA, Karunanidhi A, Mateg Ali A, Ghaznavi-Rad E, Ghasemzadeh-Moghaddam H, Chong Seng JS (2012) Prevalence of adhesion and regulation of biofilm-related genes in different clones of staphylococcus aureus. J Biomed Biotechnol 2012: 976972.

Bertschinger HU, Moon HW, Whipp SC (1972) Association of Escherichia coli with the small intestinal epithelium. I. Comparison of enteropathogenic and nonenteropatho-genic porcine strains in pigs. Infect Immun 5: 595-605.

Candela M, Perna F, Carnevali P, Vitali B, Ciati R, Gionchetti P, Rizzello F, Campieri M, Brigidi P (2008) Interaction of probiotic Lactobacillus and Bifidobacterium strains with human intestinal epithelial cells: adhesion properties, competition against enteropathogens and 
modulation of IL-8 production. Int $\mathbf{J}$ Food Microbiol 125: 286-292.

Hohmann A, Wilson MR (1975) Adherence of enteropathogenic Escherichia coli to intestinal epithelium in vivo. Infect Immun 12: 866-880.

Imberechts H, Bertschinger HU, Stamm M, Sydler T, Pohl P, De Greve H, Hernalsteens JP, Van Montgu M, Lintermans P (1994) Prevalence of F107 fimbriae on Escherichia coli isolated from pigs with oedema disease or post-weaning diarrhoea. Vet Microbiol 40: 219-230.

Li X, Deng LX, Wu GX, Cao Y, Fan B, Zhao J, Han Q, Chen SZ, Gao X, Huang N (2010) Effects of HMGN2 on bacterial adhesion to Hela Cells. Medical Journal of West China 22: 788-792.

Livak KJ, Schmittgen TD (2001) Analysis of relative gene expression data using real-time quantitative PCR and the $2^{-\Delta \Delta C T}$ Method. Methods 25: 402-408.

Melkebeek V, Rasschaert K, Bellot P, Tilleman K, Favoreel H, Deforce D, De Geest BG, Goddeeris BM, Cox E (2012) Targeting aminopeptidase N, a newly identified receptor for F4ac fimbriae, enhances the intestinal mucosal immune response. Mucosal Immunol 5: 635-645.

Nataro JP, Kaper JB (1998) Diarrheagenic Escherichia coli. Clin Microbiol Rev 11: 142-201.

Schmittgen TD (2001) Real-time quantitative PCR. Methods 25: 383-385.

Shi XH, YM, Zhou X, Chen X, Fan DL (2015) Relationship of surface properties of silicone rubber with bacteria adhesion. J Third Mil Med Univ 37: 1921-1925.

Tawakoli PN, Al-Ahmad A, Hoth-Hannig W, Hannig M, Hannig C (2013) Comparison of different live/dead stainings for detection and quantification of adherent micro- organisms in the initial oral biofilm. Clin Oral Investig 17: 841-850.

Turner SM, Scott-Tucker A, Cooper LM, Henderson IR (2006) Weapons of mass destruction: virulence factors of the global killer enterotoxigenic Escherichia coli. FEMS Microbiol Lett 263: 10-20.

Wall SJ, Edward DR (2002) Quantitative reverse transcription-polymerase chain reaction (RT-PCR): A comparison of primer-dropping, competitive, and real-time RT-PCRs. Anal Biochem 300: 269-273.

Wu SL, Yuan ZW, Ju HP, Huang XG, Hua JD, Zhou GY, Wang JY, Xie KZ, Chen GH (2007) Polymorphisms of the FUT1 gene M307 locus in post-weaning Sutai breed piglet and resistance to F18 fimbrial Escherichia coli in vitro. Chinese Journal of Preventive Veterinary Medicine 29: 783-787.

Wu ZC, Dong WH, Liu Y, Yang JS, Zhu GQ, Wu SL, Bao WB (2014) Attak Experiment and Phenotype Analysis of Meishan Piglets by E. coli F18 Strain. Acta Veterinaria Et Zootechnica Sinica 45: 1608-1615.

Yu L, Wu J, Zhu GQ (2012) Heat-labile enterotoxin enhances etec adherence to porcine small intestine epithelial cell line IPEC-J2. Chinese Journal of Animal Infectious Diseases 20: 29-35.

Zhang HY, Wang XB, Zhao XM, Wang YB, Chen LY, Xia PA, Cui BA (2010) Radix Isatis Polysaccharides to Inhibit Adhesion of Pathogenic E. coli to Cell. J Microbiol 30: 61-63.

Zhang W, Zhao M, Ruesch L, Omot A, Francis D (2007) Prevalence of virulence genes in Escherichia coli strains recently isolated from young pigs with diarrhea in the US. Vet Microbiol 123: 145-152. 